\title{
Yiqihuoxue decoction protects against post-myocardial infarction injury via activation of cardiomyocytes PGC-1a expression
}

Fanghe Li, Shuwen Guo*, Chunguo Wang, Xiaolou Huang, Hui Wang, Xiaobo Tan, Qian Cai, Jiani Wu, Yuqin Zhang, Xi Chen, Wangou Lin and Binyue Zhang

\begin{abstract}
Background: Mitochondrial dysfunction has been implicated in the pathogenesis of ischemic heart disease, exacerbating cardiomyocytes injury in myocardial infarction (MI). Peroxisome proliferator-activated receptor gamma co-activator (PGC-1a) has been recognized as the key regulator of mitochondrial biogenesis and energy metabolism. Yiqihuoxue decoction (YQHX), a Traditional Chinese Medicine (TCM) prescription, can prevent and treat ischemic heart disease. However, the mechanisms of YQHX on PGC-1 a expression in the ischemic heart have remained unclear.

Methods: Myocardial ischemia rat model and ischemia/hypoxia injury model in the cardiomyocytes were used to minic human cardiovascular disease. Rats were randomly assigned into 4 groups: Sham, Model, YQHX (8.2 g/kg) and Trimetazidine (10 mg/kg) group. 28 days after Ml, cardiac functions and morphology were detected by echocardiography and HE staining, respectively. In vitro, the effects of YQHX on H9c2 cell viability, LDH and ROS were detected, respectively. PGC-1a relevant proteins were evaluated by Western blotting.

Results: In vivo, echocardiography and HE staining results showed that YQHX improved cardiac functions and modified pathological changes. YQHX enhanced PGC-1a expression and improved the mitochondrial ultrastructure and functions in rats $\mathrm{Ml}$ model for 4 weeks. Further, we explored its potential mechanisms in cardiomyocytes. In vitro, YQHX significantly enhanced cell viability and reduced LDH release and ROS production induced by hypoxia in cardiomyocytes. Interestingly, exposure of cardiomyocytes to hypoxic conditions for $12 \mathrm{~h}$ induced the downregulation of PGC-1a expression, but the expression levels nearly returned to the normal state after hypoxia for $24 \mathrm{~h}$. YQHX significantly enhanced PGC-1a expression between $12 \mathrm{~h}$ and $24 \mathrm{~h}$ induced by hypoxia through a mechanism associated with the activation of AMPK phosphorylation in H9c2 cells. In addition, YQHX upregulated the expression of Tfam and NRF-1, while NRF-1 expression was completely blocked by an AMPK inhibitor. YQHX largely restored the mitochondrial morphology and increased mitochondrial membrane potential in hypoxia-induced injury. Furthermore, the UHPLC-LTQ-Orbitrap-MS ${ }^{n}$ analysis found that there were 87 chemical constituents in YQHX.
\end{abstract}

Conclusions: These results suggest that the protective effect of YQHX on cardiomyocytes against hypoxia-induced injury may be attributed to activation of PGC-1a and maintenance of mitochondrial functions through a mechanism involving the activation of AMPK phosphorylation.

Keywords: Yiqihuoxue decoction (YQHX), Myocardial ischemia, Cardiomyocytes, PGC-1a

\footnotetext{
* Correspondence: guo1163@163.com

Beijing University of Chinese Medicine, Beijing 100029, China

(c) The Author(s). 2018 Open Access This article is distributed under the terms of the Creative Commons Attribution 4.0 International License (http://creativecommons.org/licenses/by/4.0/) which permits unrestricted use, distribution, and reproduction in any medium, provided you give appropriate credit to the original author(s) and the source, provide a link to the Creative Commons license, and indicate if changes were made. The Creative Commons Public Domain Dedication waiver (http://creativecommons.org/publicdomain/zero/1.0/) applies to the data made available in this article, unless otherwise stated.
} 


\section{Background}

Ischemic heart disease (IHD), a crucial common cardiovascular disease, is characterized by reduced blood supply to the heart which leads to cardiomyocytes loss, ventricular remodeling, and even heart failure [1]. IHD is a public health problem with high morbidity and mortality [2]. Indeed, epidemiological studies have reported that IHD ranks as the second leading causes of death in China in 2016 [3]. Myocardial infarction (MI) is the primary pathologic complication in IHD caused by coronary artery obstruction. Although the rapid restoration of coronary artery blood flow can limit the infarct size and eventually improve the survival of MI patients [4, 5], this intervention does not adequately prevent the deterioration of cardiac function as it is associated with reperfusion injury. Therefore, additional research is required to develop efficient therapeutic options for the clinical treatment of IHD.

It is known that the mitochondria are the powerhouses of cardiomyocytes. After myocardial infarction, a series of mitochondrial alterations occur in the heart, including alterations in mitochondrial morphology and biogenesis [6], excessive accumulation of reactive oxygen species (ROS) [7] and the opening of the mitochondrial membrane potential [8]. Therapeutic measures targeting the mitochondria have been recognized to prevent and treat the cardiovascular diseases. Peroxisome proliferator-activated receptor gamma (PPAR $\gamma$ ) co-activator (PGC-1 $\alpha)$, a member of the PGC-1 family [9], has been recognized as a central regulator of mitochondrial biogenesis and energy metabolism [10]. PGC-1 $\alpha$ is a regulator of mitochondrial biogenesis via activating nuclear respiratory factor 1 (NRF-1) and the synthesis of the transcription factor A mitochondrial (Tfam) [11]. The PGC- $1 \alpha$ activity can be modulated by AMP-activated protein kinase (AMPK) phosphorylation [12]. It has also been associated with a wide range of biological processes. There is increasing evidence that PGC- $1 \alpha$ exerts different effects on cell metabolism depending on the type of organs. For instance, Aatsinki et al. [13] reported that PGC-1 $\alpha$ activated gluconeogenesis in hepatocytes, while Ye et al. [14] showed that PGC-1 $\alpha$ prevented hypoxia-induced pulmonary artery endothelial dysfunction. Thus, there has been substantial interest to understand how PGC-1 $\alpha$ becomes downregulated during myocardial infarction.

Yiqihuoxue Decoction (YQHX) is designed based on the well-known Traditional Chinese Medicines (TCMs) formula Danggui Buxue decoction (DBD). DBD, consisting of Astragalus membranaceus and Angelica sinensis, has been first described in 1247 A.D. by Dong-Yuan Li in < Neiwaishangbianhuolun $>$ [15]. DBD has been used for nourishing "qi" and enriching "blood" to prevent and treat cardiovascular diseases such as myocardial infarction [16, 17]. Compound Astragalus and Angelica extract also were found to promote angiogenesis via regulating
VEGFR1/2 and SEGFR1/2 expressions in myocardial infarction rat [16]. Based on the theory of tonifying "qi" and activating "blood" using Danggui Buxue decoction, YQHX is widely used in the prevention and treatment of cardiovascular disease by promoting angiogenesis, inhibiting inflammatory response, and regulating left ventricular function and energy metabolism [18-21]. In myocardial infarction rats model, our previous studies showed that YQHX promoted angiogenesis by the upregulation of vascular endothelial growth factor expression after 28 days [19]. YQHX was shown to regulate the metabolic-related products such as lipids, amino acids and glycolipids using nuclear magnetic resonance metabolomics [20]. YQHX could ameliorate the cardiac energy metabolism via cross-talk between the LKB1-dependent Notch1 and AMPK after myocardial infarction [21]. Also, YQHX could prevent and treat post-MI myocardial remodeling through regulating left ventricular function and promoting the expression of AMPK signal pathway [22]. However, whether YQHX influences the expression of PGC-1 $\alpha$ in the ischemic myocardium, especially in $\mathrm{H} 9 \mathrm{c} 2$ cardiomyocytes, remains unclear. Therefore, we aimed to investigate the effects and the mechanisms of YQHX on PGC- $1 \alpha$ expression and its cardioprotective effects in vitro and in vivo. This study will provide further insight into the mechanisms of TCM in the prevention and treatment of IHD.

\section{Methods \\ YQHX preparation}

YQHX is composed of five medicinal herbs: Astragalus membranaceus, Angelica sinensis, Panax ginseng, Ligusticum wallichii, and Panax notoginseng. The herbs were purchased from the Dongzhimen Hospital in China. The crude components of YQHX were extracted by refluxing with boiling distilled water $(1: 10, \mathrm{~g} / \mathrm{mL})$, each three times. After filtration, the water extracts were concentrated to a constant volume. The concentrated extracts were used for animal experiments and were prepared in the form of powders by freeze-drying in a vacuum to be used for cell experiments. A portion of each filtrate was diluted 4-fold with distilled water and further subjected to UHPLC-LTQ-Orbitrap-MS.

\section{HPLC-linear ion trap-Orbitrap mass spectrometry}

Chromatographic separation was performed on an Agilent Zorbax SB-C $\mathrm{C}_{18}$ column $(4.6 \mathrm{~mm} \times 250 \mathrm{~mm}, 5 \mu \mathrm{m})$, and the temperature was settled at $25{ }^{\circ} \mathrm{C}$. The flow rate was $1.0 \mathrm{~mL} / \mathrm{min}$, the UV spectra were $254 \mathrm{~nm}$ and the mobile phases were a mixture of ultrapure water (A) and acetonitrile (B). The following gradient program was used: 0$10 \mathrm{~min}, 2 \% \mathrm{~B} ; 10-15 \mathrm{~min}, 2-5 \% \mathrm{~B} ; 15-52 \mathrm{~min}, 5-42 \% \mathrm{~B}$; 52-70 min, 42-90\% B; 70-75 min, 90-90\% B; 75-80 min, $90-5 \%$ B. High-resolution mass spectra were analyzed using an LTQ-Orbitrap mass spectrometer (Thermo Scientific, Bremen, Germany), which was operated in the 
negative ionization mode. The parameters were as follows: sheath gas flow rate, 30 arb; aux gas flow rate, 10 arb; capillary temperature, $350{ }^{\circ} \mathrm{C}$; ion spray voltage, $4.0 \mathrm{kV}$; capillary voltage, $-25 \mathrm{~V}$; and tube lens voltage, $-110 \mathrm{~V}$. In the full scan mode, mass spectra were recorded in the mass range of $m / z 150$ to 1200 with a resolution of 30,000 (full width at half-maximum, as defined at $\mathrm{m} / \mathrm{z} 400$ ).

\section{Animal study}

In accordance with the Guide for the Animal Care and Use of Laboratory Animals published by the National Institutes of Health (NIH Publications No. 85-23, revised 1996), all experimental protocols and animal handling procedures were approved by the Animal Care and Use Committee of Dongzhimen Hospital Affiliated to Beijing University of Chinese Medicine (2017-11). Adult male Sprague-Dawley (SD) rats (180-200 g) were obtained from Beijing-Vital-River-Laboratory-Animal Technology (license number: SCXK2016-0011) and were subjected to myocardial infarction surgery. Rats were allowed free access to regular diet and distilled water, and were housed in an air-conditioned animal room at the Key Laboratory of Dongzhimen Hospital before surgery (temperature: $21 \pm 2{ }^{\circ} \mathrm{C}$, humidity: $50 \pm 5 \%$ ). The myocardial infarction rat model was induced as described previously [20, 23, 24]. Firstly, the rats were anesthetized with $1 \%$ pentobarbital sodium $40 \mathrm{mg} / \mathrm{kg}$ by intraperitoneal injection. The chest of the rat was opened by left thoracotomy to expose the heart, and the left anterior descending (LAD) coronary artery was ligated using a 5-0 nylon suture in the MI group and then the chest was closed. The procedure was the same in the Sham group except the LAD ligation. The success of MI model was confirmed by significant elevation of the ST segment arch obtained by monitoring the electrocardiogram (ECG) limb lead (Medical electronic instrument factory, Jiangsu, China). After four weeks, the rats that survived were subjected to echocardiographic examination and those with left ventricular ejection fraction (LVEF) of less than $50 \%$ were chosen for subsequent studies. All animals were anaesthetized by pentobarbital sodium, and then cardiac samples were collected to analyze protein expression levels. After that, the rats were euthanized by cervical dislocation. The thumb and index finger of researcher was pressed at the base of the rat's skull. On the other hand, the base of the tail was rapidly pulled, causing a separation of the cervical vertebrae from the skull.

The experimental animals were randomly divided into four groups ( $n=12$, 8-week-old): Sham operation + vehicle (Sham), Myocardial infarction model + vehicle (MI), Myocardial infarction model + YQHX (YQHX), Myocardial infarction model + Trimetazidine (TMZ). The YQHX was administered at the dosage of $8.2 \mathrm{~g} / \mathrm{kg} / \mathrm{d}$ and the dosage of TMZ (Servier pharmaceutical Co., Ltd., Tianjin, China) was
$10 \mathrm{mg} / \mathrm{kg} / \mathrm{d}$ by irrigation on the second day after MI surgery, as described before [25].

\section{Cell culture and treatment}

$\mathrm{H} 9 \mathrm{c} 2$ cells were seeded and cultured in high-glucose DMEM (Invitrogen, California, USA) with $10 \%$ fetal bovine serum FBS, $1 \%$ penicillin and streptomycin in a cell incubator with $5 \% \mathrm{CO}_{2}$ and $95 \% \mathrm{O}_{2}$ at $37{ }^{\circ} \mathrm{C}$. The medium was completely replaced with serum-free DMEM. After synchronization of the culture for $6 \mathrm{~h}$, the control group (C) was cultured in a normoxic atmosphere and the ischemia/hypoxia injury model $(\mathrm{I} / \mathrm{H})$ in $\mathrm{H} 9 \mathrm{c} 2$ cells was induced by exposure of the cells to a hypoxic atmosphere with $1 \%$ $\mathrm{O}_{2}, 5 \% \mathrm{CO}_{2}$ and $94 \% \mathrm{~N}_{2}$ for $12 \mathrm{~h}$ or $24 \mathrm{~h} .25 \mathrm{mg}$ of $\mathrm{YQHX}$ were dissolved in $50 \mathrm{~mL}$ of DMEM and used for the treatment of hypoxia-induced H9c2 cells [26]. Thereafter, the YQHX extracts were filtered through a $0.45 \mu \mathrm{m}$ millipore filter prior to use. In the rescue groups, the $\mathrm{H} 9 \mathrm{c} 2$ cells were treated with $\mathrm{YQHX}$ at the concentrations of $100 \mu \mathrm{g} / \mathrm{mL}$ (Y1), $200 \mu \mathrm{g} / \mathrm{mL}$ (Y2) and $400 \mu \mathrm{g} / \mathrm{mL}(\mathrm{Y} 4)$, and Compound $\mathrm{C}(5 \mu \mathrm{M})$ (Selleck, Shanghai, China) with YQHX (Y2cc) before the initiation of hypoxia.

\section{Histologic examination and echocardiography}

Cardiomyocytes architectures were visualized using hematoxylin and eosin (HE) staining. Rat heart tissues were fixed in $4 \%$ paraformaldehyde for $24 \mathrm{~h}$ and then were embedded in paraffin. The tissue blocks were cut into $4 \mu \mathrm{m}$ sections, which were stained with $\mathrm{HE}$ to observe the morphology.

Cardiac functions such as the left ventricular internal diastolic diameter (LVIDd), and internal systolic diameter (LVIDs), ejection fraction (EF) and fractional shortening (FS) were measured by echocardiography at 4 weeks after surgery using a 30-MHz high-resolution Vevo 770 smallanimal ultrasonic instrument probe (Visual Sonics Inc., Toronto, Canada).

\section{Measurement of ATP levels}

The fresh samples were homogenized in cold lysis solution for $30 \mathrm{~min}$. The lysate was centrifuged at $12,000 \mathrm{~g}$ and $4{ }^{\circ} \mathrm{C}$ for $5 \mathrm{~min}$ to obtain the supernatant. The ATP levels were detected by ATP Assay Kits (Beyotime, Shanghai, China) according to the manufacturer's protocol.

\section{Cell viability assay}

H9c2 cells were seeded and cultured in 96-well plates. Cell viability was examined using CCK8 assay kit. H9c2 cells were incubated with the cell counting kit-8 (CCK8, Dojindo Laboratories, Kyushu, Japan) for $2 \mathrm{~h}$ at $37{ }^{\circ} \mathrm{C}$ and then cell viability was measured at $450 \mathrm{~nm}$ by a microplate reader. The cell viability was calculated as the percentage of absorbance to control values. 


\section{LDH assay}

To further assess the cell injury, the release of lactate dehydrogenase (LDH) was tested by LDH assay. The level of LDH in H9c2 cell supernatants was measured using LDH commercial kit, according to the manufacturer's protocol (Jian Cheng Bioengineering Institute, Nanjing, China). LDH value was examined using a microplate reader at $440 \mathrm{~nm}$ and was expressed as U/L.

\section{ROS measurement}

H9c2 cells were washed three times with phosphate buffered saline (PBS), and then incubated with PBS and $10 \mu \mathrm{M}$ fluorescent probe $2^{\prime}, 7^{\prime}$-dichlorofluorescein diacetate (DCFH-DA) in the dark for $30 \mathrm{~min}$ at $37^{\circ} \mathrm{C}$. Then the level of ROS was estimated by fluorescence microscope (Nikon, Tokyo, Japan).

\section{Transmission electron microscopy}

Fresh heart tissues in the margin area of the infarct were cut into less than $1 \mathrm{~mm}^{3}$ cubes and rapidly fixed with $2 \%$ paraformaldehyde and $2.5 \%$ glutaraldehyde at $4{ }^{\circ} \mathrm{C}$ for $2 \mathrm{~h}$. The fixed samples were washed three times with PBS. Similarly, H9c2 cells were washed and then fixed by $2 \%$ paraformaldehyde and $2.5 \%$ glutaraldehyde at $4{ }^{\circ} \mathrm{C}$ for $2 \mathrm{~h}$. The cells were collected by gentle scratching gently with a cell scraper. After washed three times, cardiac samples and H9c2 cells were further processed. Copper grids were stained by $2 \%$ uranyl acetate, and then embedded with epoxy resin and heated to $60{ }^{\circ} \mathrm{C}$ to allow polymerization. Images were recorded by transmission electron microscope (Hitachi-H7650, Tokyo, Japan).

\section{Mitochondrial membrane potential $\left(\Delta \Psi_{\mathrm{m}}\right)$}

$\mathrm{H} 9 \mathrm{c} 2$ cells were seeded at $1 \times 10^{5}$ cells $/ \mathrm{mL}$ in a confocal dish. As described above, $\mathrm{H} 9 \mathrm{c} 2$ cells were incubated with $5 \mu \mathrm{M}$ JC-1 probe (Beyotime, Shanghai, China). The depolarization ratio of $\Delta \Psi \mathrm{m}$ was determined at an excitation wavelength of $490 \mathrm{~nm}$ and $525 \mathrm{~nm}$ as JC-1 monomer (green fluorescence) and emission wavelengths of $530 \mathrm{~nm}$ and $590 \mathrm{~nm}$ as JC-1 aggregate (red fluorescence) in the confocal laser scanning microscopy (Olympus, Tokyo, Japan).

\section{Real-time quantitative PCR analysis}

Total RNA was extracted from H9c2 cells using Trizol reagent (Invitrogen, California, USA) and subjected to reverse transcription. Thereafter, the first cDNA was synthesized using a Revert Aid First Strand cDNA Synthesis kit (Invitrogen, California, USA). Real-time quantitative PCR was carried out using SYBR Premix Ex Taq kit on an ABI PRISM 7500 PCR instrument (Applied Biosystems, New York, USA). The cDNA was denatured by 35 PCR cycles $\left(94{ }^{\circ} \mathrm{C}, 2 \mathrm{~min} ; 94{ }^{\circ} \mathrm{C}, 30 \mathrm{~s} ; 61^{\circ} \mathrm{C}, 30 \mathrm{~s} ; 72{ }^{\circ} \mathrm{C}, 30 \mathrm{~s}\right)$. GAPDH was the invariant control, and the relative level of
mRNA was analyzed by the $2^{-\Delta \Delta C t}$ method. The sequences for the primers used are listed: PGC-1 $\alpha$ : forward, 5'-AGC CAC TAC AGA CAC CGC AC-3' and reverse, 5'-CCT TTC AGA CTC CCG CTT C-3' and GAPDH: forward, 5'-GGC AAG TTC AAC GGC ACA G-3' and reverse, 5'-GCC AGT AGA CTC CAC GAC AT-3'.

\section{Western blotting}

Samples of myocardial tissues from the margin area of the infract were prepared for protein analysis. The heart tissues samples were homogenized in RIPA lysis buffer containing a protease inhibitor cocktail. The same procedures were used for the H9c2 cells. H9c2 cells were collected by scraping with ice-cold RIPA lysis buffer, and the proteins were quantified using BCA protein assay kit. Cell lysates ( $40 \mu \mathrm{g}$ of protein) were separated with $12 \%$ SDSPAGE blots and then transferred to the membranes (Millipore, USA). These membranes were blocked with 5\% fat-free milk for $2 \mathrm{~h}$ and incubated with the following primary antibodies: PGC-1 $\alpha$ (Abcam, Cambridge, England), p-AMPK (Cell Signaling Technology, New England, USA), NRF-1 (Abcam, Cambridge, England) and Tfam (Abcam, Cambridge, England) at $4{ }^{\circ} \mathrm{C}$ overnight. $\beta$-actin was used as a loading control. After washing, the polyvinylidene fluoride membranes were incubated with secondary antibodies (goat anti-rabbit IgG 1:5000 and goat anti-mouse IgG 1:5000) for $2 \mathrm{~h}$. Blots were visualized using with an enhanced chemiluminescence (ECL) detection kit (PIERCE, Massachusetts, USA) and exposure to the X-ray film.

\section{Statistical analysis}

The data were presented as mean \pm SD. SPSS 17.0 software (IBM, Armonk, USA) was used for statistical analysis. Student's t-test or one-way or repeated-measures ANOVA were used to compare values between groups, and $P$ less than 0.05 was considered as statistically significant. The analyses were performed by GraphPad Prism software. Xcalibur 2.1 software (Thermo-Fisher Scientific, Bremen, Germany) was used for the qualitative characterization of chemical constituents.

\section{Results}

Identification of 87 chemical constituents in YQHX

YQHX sample was analyzed with rapid separation using the optimized LC-ESI-MS ${ }^{\mathrm{n}}$ method. The total ion chromatogram (TIC) of YQHX sample in negative ionization mode was shown in Fig. 1, and there were 87 peaks in YQHX preparations (Fig. 1 and Additional file 1). For most of the constituents in YQHX, $(\mathrm{M}-\mathrm{H})^{-}$and $(\mathrm{M}+$ $\mathrm{COOH})^{-}$ions were listed in Additional file 1 and consisted of triterpenoid saponins and flavonoids. These data provided valuable information, on the molecular weights and structure of the constituents. 


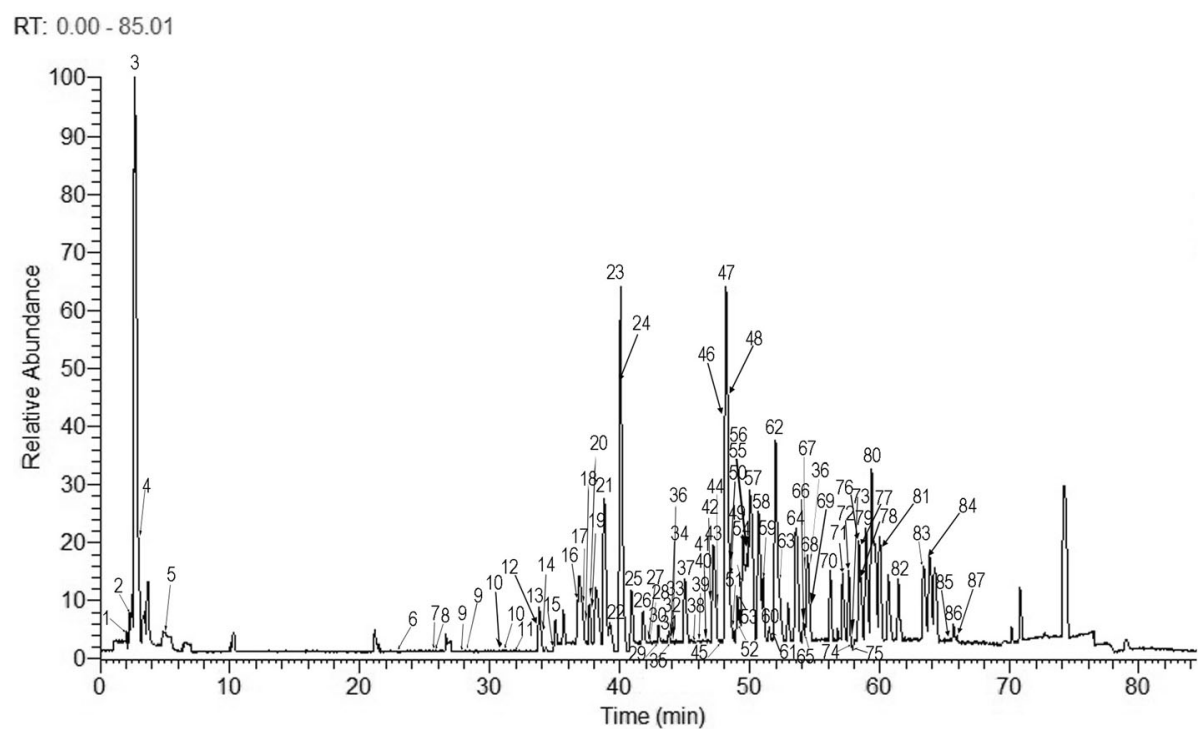

Fig. 1 The total ion chromatogram of YQHX obtained in negative ionization mode based on UHPLC-LTQ-Orbitrap-MS

Taking ginsenoside $\mathrm{Rb} 1$ as an example, the structure of chemical components in YQHX was as follows: product 47 (peak 2 in the Fig. 1) displayed quasi-molecular ion peak at $\mathrm{m} / z 1153.59875(\mathrm{M}+\mathrm{COOH})^{-}$and $1107.59558(\mathrm{M}-\mathrm{H})^{-}$ with retention time of $48.13 \mathrm{~min}$ and characteristic fragment ions at $m / z$ 945, $m / z 783, m / z 621, m / z 459$ (Fig. 2a), which showed the typical fragmentation characteristics of ginsenoside $\mathrm{Rb} 1$. According to the composition analysis, the molecular formula of the compound was $\mathrm{C}_{54} \mathrm{H}_{92} \mathrm{O}_{23}$ (errors within $1.12 \mathrm{ppm}$ ). The fragment ions were produced by the loss of a molecule of glucose, two molecules of glucose, three molecules of glucose, and four molecules of glucose $\left(m / z 945\right.$ (MH-Glc) $^{-}, m / z 621$ (MH-3Glc) $^{-}$and $m / z$ $\left.459(\mathrm{MH}-4 \mathrm{Glc})^{-}\right)$, respectively. Therefore, it was identified as ginsenoside $\mathrm{Rb} 1$, and the mass spectral fragmentation pathways of ginsenoside $\mathrm{Rb} 1$ are shown in Fig. $2 \mathrm{~b}$. The structures of the other 86 constituents in YQHX were obtained according to the above method.

\section{YQHX improved cardiac dysfunction and enhanced PGC- 1a expression in $\mathrm{Ml}$ rat hearts}

To determine the effects of YQHX on cardiomyocyte architecture and cardiac function after MI injury, echocardiography analysis showed that YQHX and TMZ significantly improved cardiac dysfunction and reversed cardiac remodeling in MI heart after 4 weeks (Fig. 3a). Specifically, echocardiography showed that YQHX treated groups had a significantly higher LVEF and LVFS, and remarkably lower LVIDs compared with the MI group. However, there was no significant difference in LVIDd between YQHX group and MI group. As shown in Fig. 3b, we observed a high number of inflammatory cell infiltration, enlargement of the intercellular space and extensive edema of cardiomyocytes in rats 4 weeks after MI injury compared to the Sham group. After treatment with YQHX and TMZ, there were numerous changes in the myocardium of rats, including slight inflammatory cell infiltrates and mild edema of cardiomyocytes. Next, we investigated whether YQHX and TMZ affected PGC- $1 \alpha$ expression in a rat model of myocardial infarction. TMZ, a pharmacological inhibitor of thiolase and an enzyme involved in a key step of $\beta$-oxidation, has been reported to protect against ischemia/reperfusion injury [23]. As shown in Fig. 3c, PGC-1 $\alpha$ protein expression in MI group was significantly lower compared with that of Sham group, while YQHX markedly enhanced PGC- $1 \alpha$ expression $(P<0.05)$. However, TMZ failed to increase PGC- $1 \alpha$ protein expression.

YQHX protected against Ml-induced mitochondrial dysfunction The sustained energy requirements of the heart require high daily consumption of ATP to sustain the continuous contractile process of the heart [24]. The mitochondria comprise $23 \%$ to $32 \%$ of the myocellular volume and generate about $90 \%$ of the required ATP $[24,25]$. We investigated whether the protective effect of YQHX is linked to the mitochondrial functions in rats MI model. As shown in Fig. 4a, the ultrastructure of the myocardium in the Sham group was normal and cardiac muscle fibers were regularly arranged. After MI injury, the myocardial ultrastructure was irregular and that of the mitochondria was disrupted as illustrated by the swelling, rupture, vacuoles and loss of cristae. Interestingly, YQHX treatment improved myocardial ultrastructure during injury and relatively enhanced the proliferation and accumulation of mitochondria. Moreover, chronic myocardial infarction 


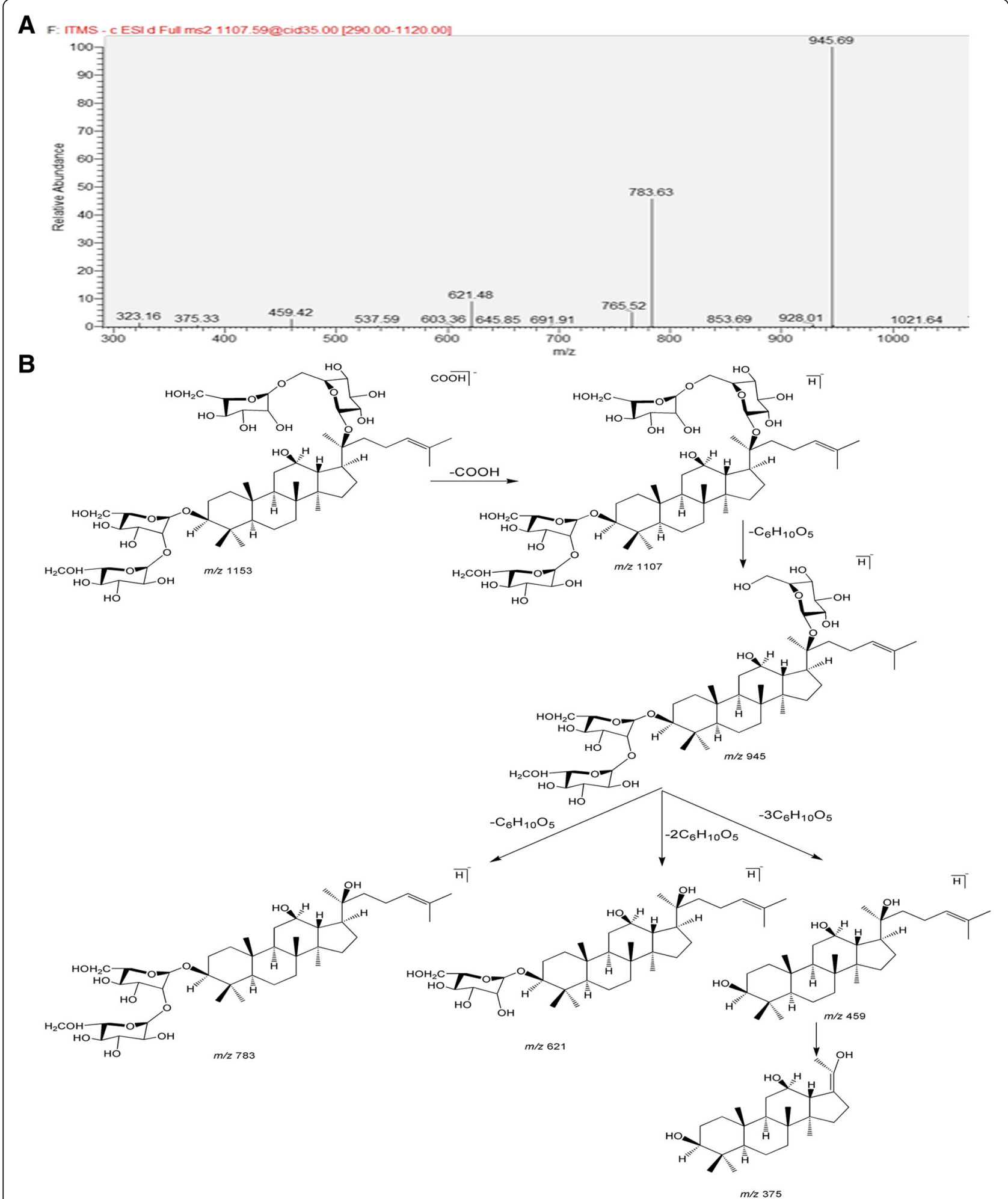

Fig. 2 Identification of ginsenoside Rb1 in YQHX preparations. a The characteristic fragment ions of ginsenoside Rb1 at $m / z$ 945, 783, 621, and 459. $\mathbf{b}$ The mass spectral fragmentation pathways of ginsenoside Rb1 


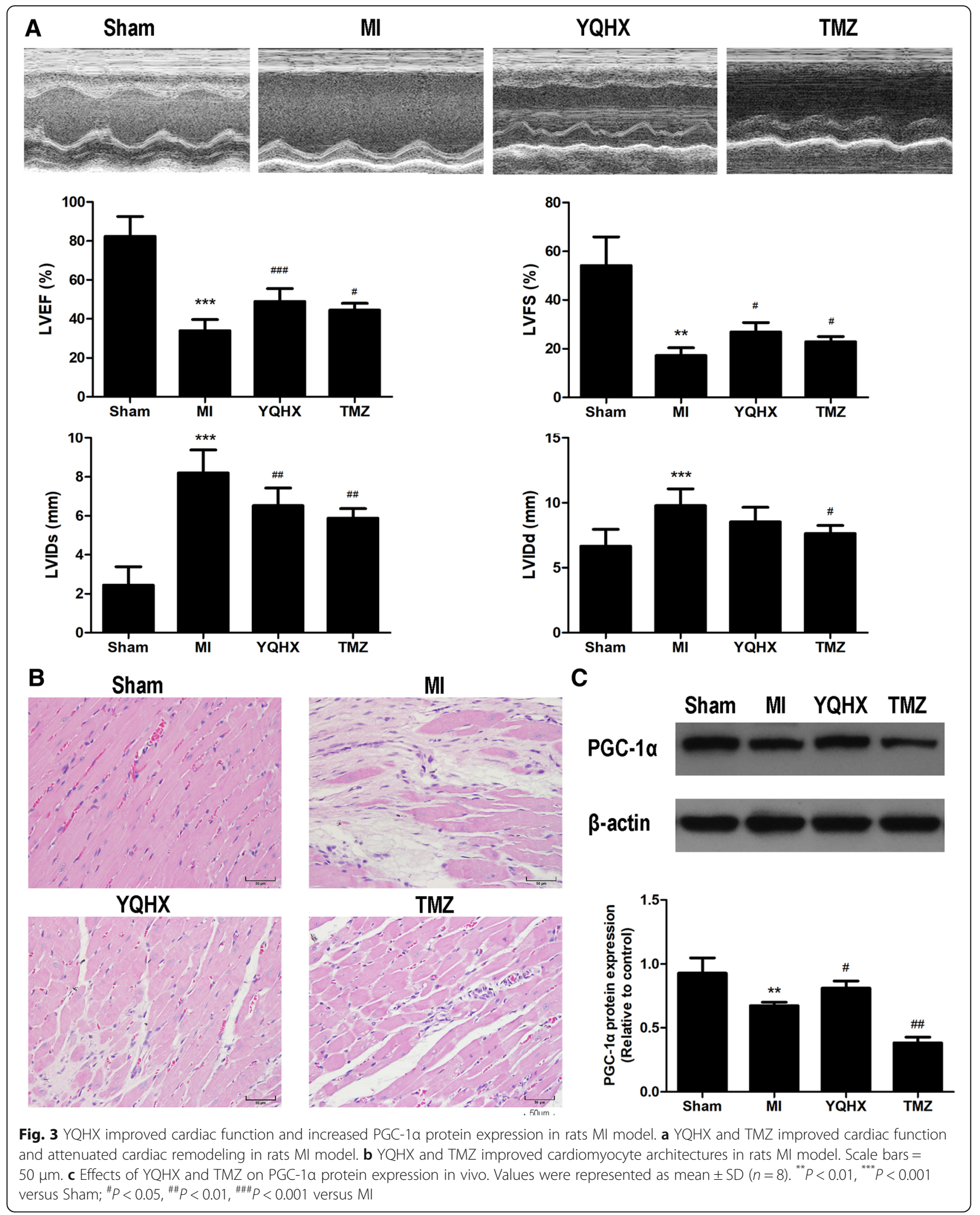



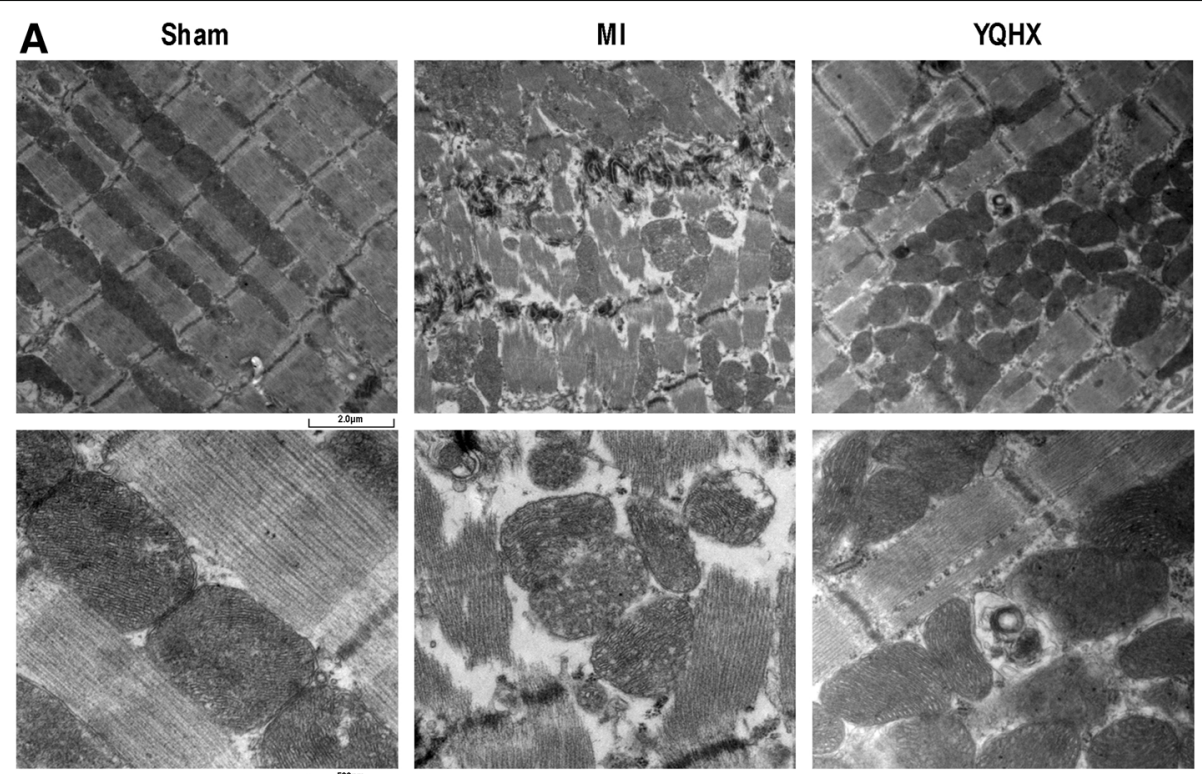

B

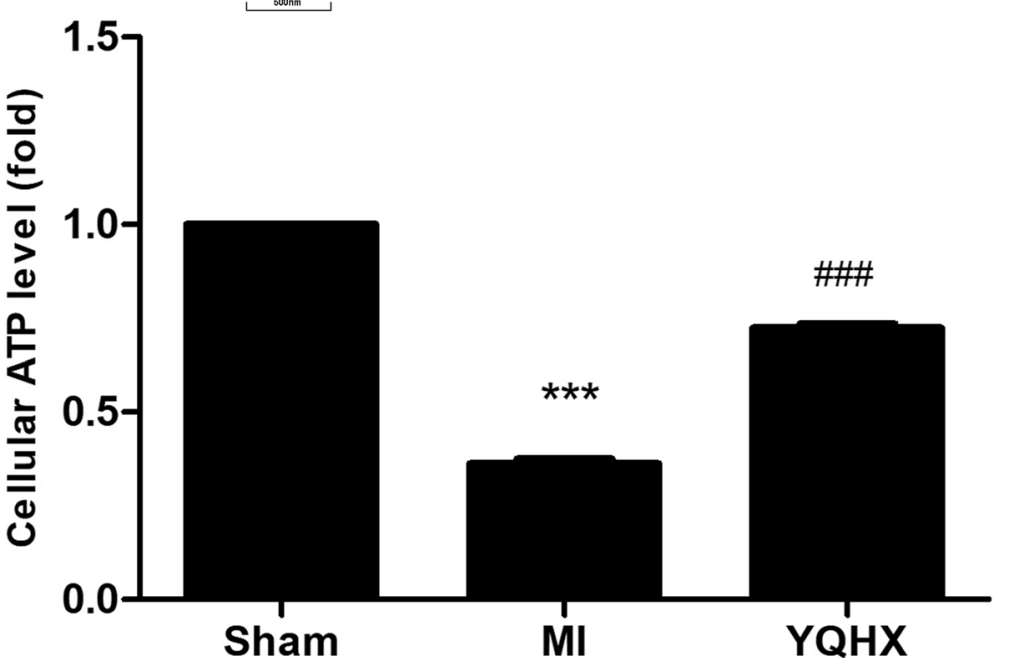

Fig. 4 YQHX treatment improved myocardial ultrastructure and mitochondrial function in myocardial infarction rat model. a Effect of YQHX treatment on the myocardial ultrastructure in myocardial infarction rat model. Scale bars $=2.0 \mu \mathrm{m}$; Scale bars $=500 \mathrm{~nm}$. $\mathbf{b}$ Effects of YQHX treatment on mitochondrial function in Ml rat model. Values were represented as mean $\pm \operatorname{SD}(n=5) .{ }^{* * *} P<0.001$ versus Sham; ${ }^{\# \# \#} P<0.001$ versus MI

decreased the production of ATP in rats, but YQHX increased ATP production, indicating that YQHX could protect against MI-induced mitochondrial dysfunction (Fig. 4b).

YQHX enhanced cell viability of H9c2 cells cultivated in normal or ischemic/hypoxic conditions

Based on our previous research [25], YQHX at concentrations of $100-400 \mu \mathrm{g} / \mathrm{mL}$ remarkably increased cell viability induced by hypoxia for $24 \mathrm{~h}$. These concentrations were used in this study for YQHX treatment in normal or hypoxic conditions. Using the CCK8 assay, we found that YQHX significantly enhanced the cell proliferation rate at $12 \mathrm{~h}, 24 \mathrm{~h}$ or $48 \mathrm{~h}$ when cells were incubated in normal conditions (Fig. 5a). As shown in Fig. 5b, cell viability of $\mathrm{I} / \mathrm{H}$ group decreased in a time-dependent manner compared to the control group at $12 \mathrm{~h}, 24 \mathrm{~h}, 36 \mathrm{~h}$ or $48 \mathrm{~h}, \mathrm{YQHX}$ at the concentrations of $100-400 \mu \mathrm{g} / \mathrm{mL}$ reversed $\mathrm{I} / \mathrm{H}$-induced cell death and significantly increased cell viability at $12 \mathrm{~h}, 24 \mathrm{~h}$ or $36 \mathrm{~h}$. Therefore, the $12 \mathrm{~h}$ and $24 \mathrm{~h}$ time points were selected for further study.

YQHX reduced LDH levels induced by hypoxia in H9c2 cells To further assess the cell injury, LDH leakage was tested by $\mathrm{LDH}$ assay. The release of $\mathrm{LDH}$ in the hypoxia group was significantly elevated compared to the control group at $12 \mathrm{~h}, \mathrm{YQHX}$ treatment significantly reduced the level of LDH (Fig. 6). 
A

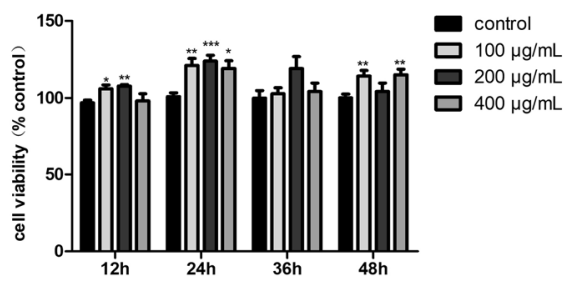

B

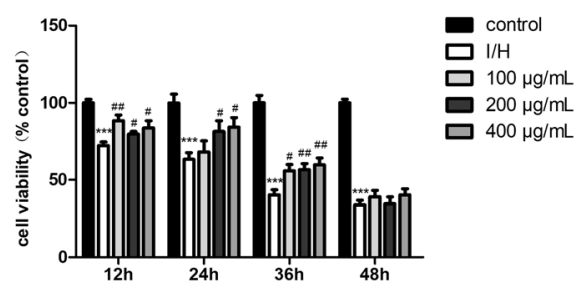

Fig. $5 \mathrm{YQHX}$ protected against injury induced by ischemia/hypoxia in H9c2 cardiomyocytes. a Effects of $\mathrm{YQHX}$ (100-400 $\mu \mathrm{g} / \mathrm{mL}$ ) on H9c2 cell viability at different time-points in normal conditions. b Effects of $Y \mathrm{QHX}(100-400 \mu \mathrm{g} / \mathrm{mL})$ treatment on $\mathrm{H} 9 \mathrm{c} 2 \mathrm{cell}$ viability subjected to $\mathrm{l} / \mathrm{H}$ at different time-points. Values were represented as mean $\pm \mathrm{SD}(n=6)$. ${ }^{*} P<0.05,{ }^{* *} P<0.01,{ }^{* * *} P<0.001$ versus control; ${ }^{\#} P<0.05$, ${ }^{\# \#} P<0.01$ versus hypoxia

\section{YQHX improved mitochondrial ultrastructure and} reversed the mitochondrial depolarization

Next, we investigated whether YQHX affected mitochondrial ultrastructures in hypoxia-treated $\mathrm{H} 9 \mathrm{c} 2$ cells. As shown in Fig. 7a, hypoxia-induced changes in the mitochondrial morphology, including swelling, black matrix and loss of cristae compared to the control. In contrast, YQHX treatment improved the mitochondrial ultrastructures in $\mathrm{I} / \mathrm{H}$-induced $\mathrm{H} 9 \mathrm{c} 2$ cells.

To further confirm the effect of YQHX on mitochondrial activity, mitochondrial membrane potential $(\Delta \Psi \mathrm{m})$ was observed by confocal laser scanning microscopy in $\mathrm{H} 9 \mathrm{c} 2$ cells. $\Delta \Psi \mathrm{m}$ was expressed as the fluorescence ratio of red to green. A low ratio represented mitochondrial depolarization. As shown in Fig. $7 \mathrm{~b}, \Delta \Psi \mathrm{m}$ of hypoxia group had a lower ratio than that of the control group, whereas $100-400 \mu \mathrm{g} / \mathrm{mL}$ YQHX treatment reversed the mitochondrial depolarization, especially at the concentration of $200 \mu \mathrm{g} / \mathrm{mL}$. These data sets indicate that YQHX at the concentration of $200 \mu \mathrm{g} / \mathrm{mL}$ was optimal in protecting the mitochondrial function hypoxia-induced injury in H9c2 cells. Based on these results, the concentration of

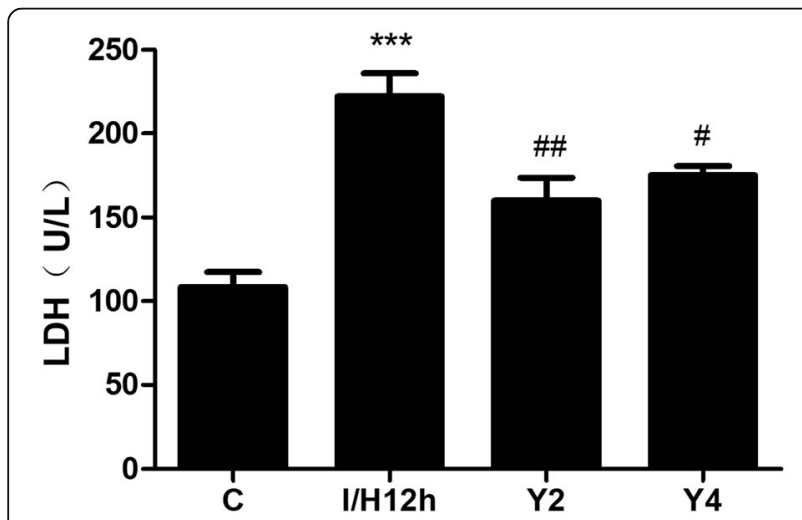

Fig. $6 \mathrm{YQHX}$ reversed the level of $\mathrm{LDH}$ in $\mathrm{H} 9 \mathrm{c} 2$ cells supernatants induced by hypoxia for $12 \mathrm{~h}$. Results were presented as mean $\pm \mathrm{SD}$ $(n=3) .{ }^{* * *} P<0.001$ versus control; ${ }^{\#} P<0.05,{ }^{\# \#} P<0.01$ versus hypoxia
$200 \mu \mathrm{g} / \mathrm{mL}$ was chosen for the application of YQHX in the present study.

\section{YQHX increased hypoxia-induced PGC-1a expression in H9c2 cells}

We further sought to determine whether hypoxia affected PGC- $1 \alpha$ mRNA and protein expression in H9c2 cells. We found that the mRNA and protein levels of PGC-1 $\alpha$ were significantly decreased following hypoxia for $12 \mathrm{~h}$ (Fig. 8a). Since PGC- $1 \alpha$ has been recognized to regulate mitochondrial dysfunction, these results were consistent with the observations that hypoxia was accompanied by mitochondria structural changes, such as black matrix and loss of cristae observed by electron transmission microscopy (Fig. 8b). We further evaluated the effects of YQHX (100-400 $\mu \mathrm{g} /$ $\mathrm{mL}$ ) on PGC- $1 \alpha$ mRNA and protein expression in hypoxia treated $\mathrm{H} 9 \mathrm{c} 2$ cells. As shown in Fig. 8c, PGC- $1 \alpha$ mRNA expression was significantly decreased in $\mathrm{I} / \mathrm{H}$-induced $\mathrm{H} 9 \mathrm{c} 2$ cells, whereas $200 \mu \mathrm{g} / \mathrm{mL}$ YQHX treatment remarkably increased the PGC- $1 \alpha$ mRNA expression $(P<0.01)$. Similar results were observed for the PGC- $1 \alpha$ protein expression in $\mathrm{H} 9 \mathrm{c} 2$ cells subjected to $12 \mathrm{~h}$ ischemic/hypoxic conditions. These in vitro results were consistent with those obtained from in vivo experiments. Mitochondria are recognized as the major cellular source of ROS. ROS overproduction results in mitochondrial abnormality. Therefore, we investigated the effect of YQHX on ROS production. As shown in Fig. 8d, the production of ROS was increased by hypoxia in H9c2 cells, and 100, 200 and $400 \mu \mathrm{g} / \mathrm{mL}$ YQHX significantly attenuated ROS overproduction.

\section{YQHX upregulated PGC-1 1 a expression induced by} hypoxia via the activation of AMPK phosphorylation Studies have demonstrated that the mRNA expression of PGC- $1 \alpha$ after exposure to hypoxic conditions for $12 \mathrm{~h}$ may differ from $24 \mathrm{~h}[14,27]$. Hence, we further examined whether exposure of H9c2 cardiomyocytes to $12 \mathrm{~h}$ and $24 \mathrm{~h}$ hypoxia affected the PGC- $1 \alpha$ expression in a similar manner. Based on the above PGC- $1 \alpha$ mRNA and protein results in hypoxia-induced $\mathrm{H} 9 \mathrm{c} 2$ for $12 \mathrm{~h}$, we 


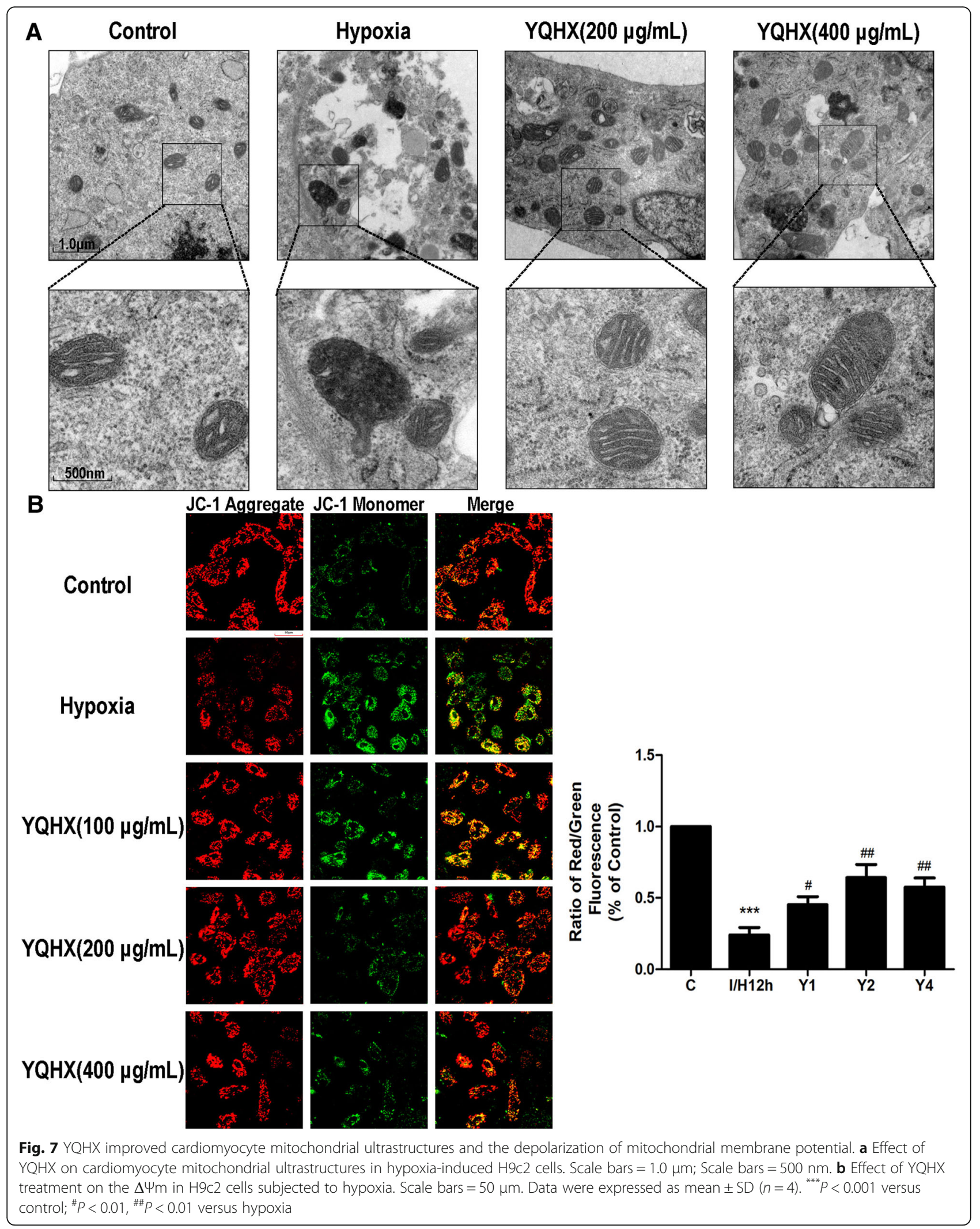




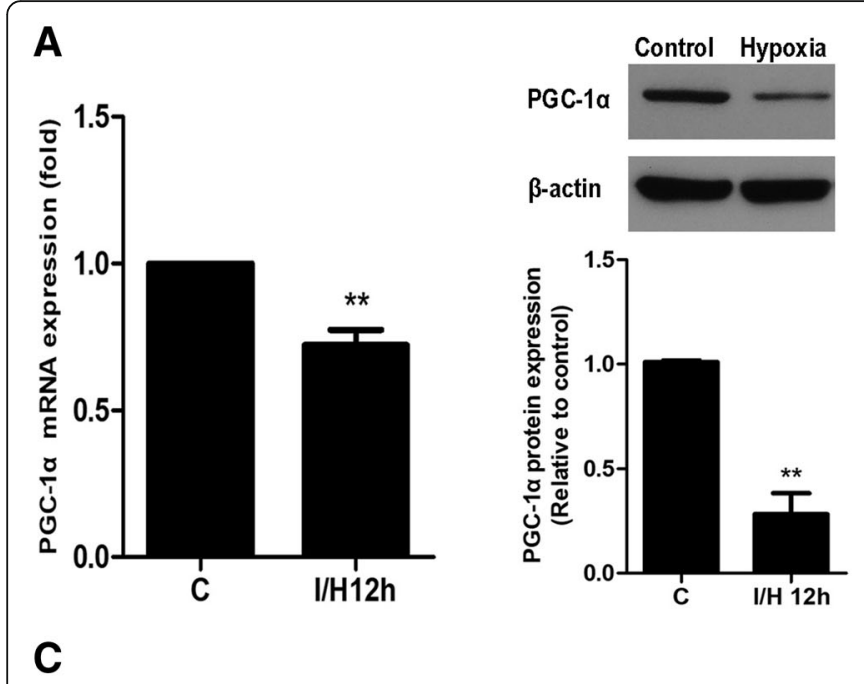

B
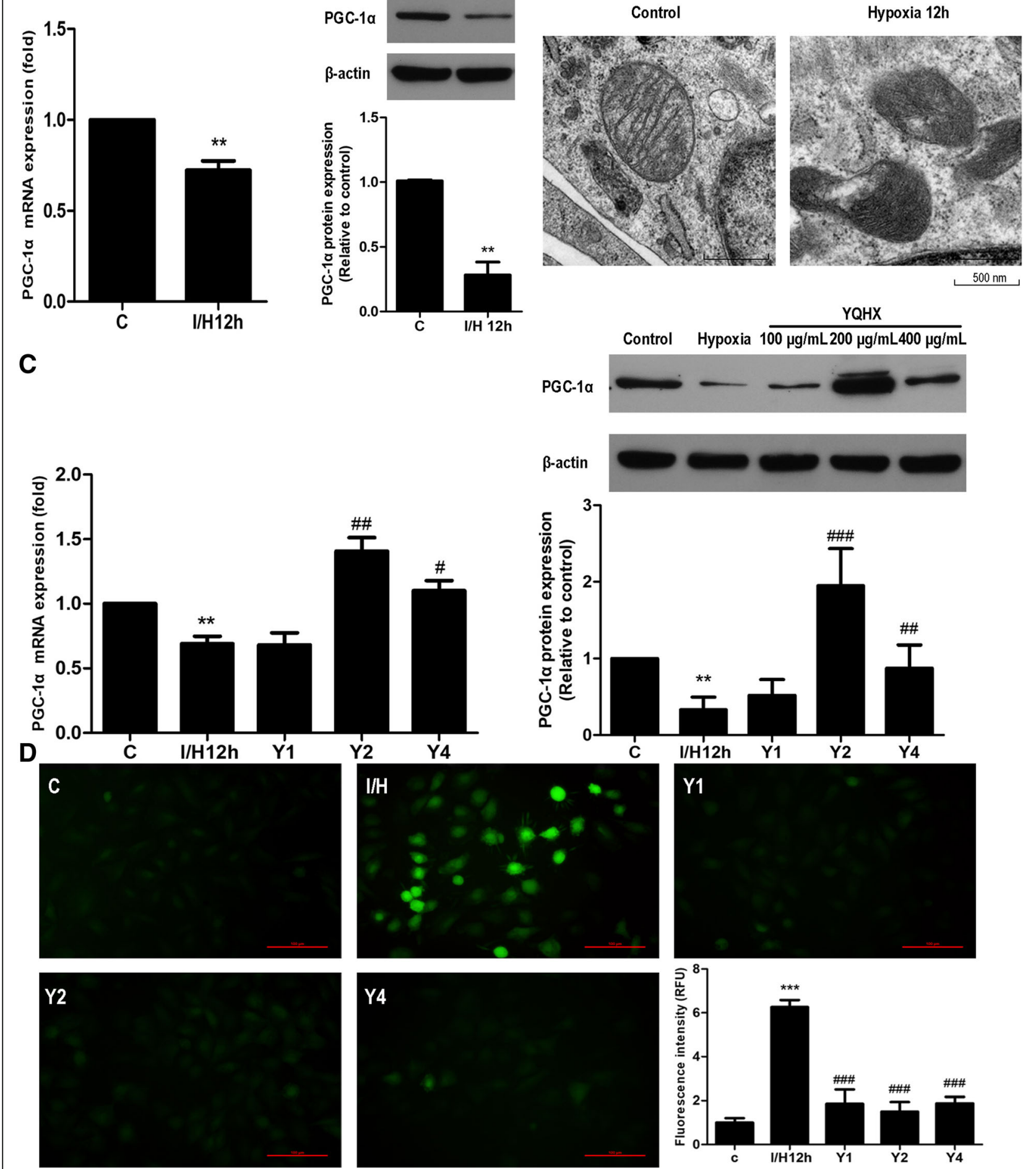

Fig. 8 YQHX enhanced PGC-1a mRNA and protein expression and inhibited ROS production in hypoxia-induced H9c2 cells for $12 \mathrm{~h}$. a Effects of ischemia/hypoxia on PGC-1 a mRNA (left) and protein (right) expression in H9c2 cells. $\mathbf{b}$ Effect of hypoxia on the mitochondrial structure in hypoxiatreated H9c2 cells. Scale bar $=500 \mathrm{~nm}$. c Effects of YQHX treatment on PGC-1a mRNA (left) and protein (right) expression in hypoxia-induced H9c2 cells. $\mathbf{d}$ Effect of $Y Q H X$ treatment on the production of ROS in $1 / \mathrm{H}$-induced H9c2 cells. Scale bar $=100 \mu \mathrm{m}$. The above results were obtained from four independent experiments. Results were presented as mean $\pm \mathrm{SD}(n=4) .{ }^{* * *} P<0.01,{ }^{* * * *} P<0.001$ versus control; ${ }^{\#} P<0.05,{ }^{\# \#} P<0.01,{ }^{\# \# \#} P<0.001$ versus hypoxia 
further extended hypoxia to $24 \mathrm{~h}$ and measured PGC- $1 \alpha$ protein expression. Interestingly, compared to the control group, PGC-1 $\alpha$ protein expression remarkably decreased at $12 \mathrm{~h}$ of hypoxia, but it nearly returned to normal level following $24 \mathrm{~h}$ (Fig. 9a). The differences in PGC-1 $\alpha$ expression between the control group and hypoxia-induced group at $24 \mathrm{~h}$ were not significant. Numerous studies have demonstrated that the upregulation of PGC- $1 \alpha$ expression may be directly regulated by AMP-activated protein kinase (AMPK) signaling [9, 14, 27, 28]. To test this possibility, we measured the phosphorylation level of AMPK in hypoxia-induced $\mathrm{H} 9 \mathrm{c} 2$ cells at $12 \mathrm{~h}$ and $24 \mathrm{~h}$. As shown in Fig. 9b, AMPK phosphorylation at threonine 172 showed an increasing trend in hypoxia-stimulated cells for $24 \mathrm{~h}$, but difference between the control group and hypoxia-induced $24 \mathrm{~h}$ group was not significant. This suggests that YQHX-induced activation of PGC- $1 \alpha$ expression may be dependent on AMPK activity. We then tested this possibility using Dorsomorphin (Compound C), an inhibitor of AMPK phosphorylation. Treatment with $5 \mu \mathrm{M}$ Compound $\mathrm{C}$ effectively suppressed PGC- $1 \alpha$ expression and completely abolished AMPK phosphorylation in hypoxia-stimulated $\mathrm{H} 9 \mathrm{c} 2$ cells at $12 \mathrm{~h}$ and $24 \mathrm{~h}$ (Fig. 9c-d). These data agreed with the results of Ye et al. [14, 26, 27]. Moreover, treatment with YQHX enhanced PGC-1 $\alpha$ expression and AMPK phosphorylation in hypoxia-induced H9c2 cells for $12 \mathrm{~h}$ and $24 \mathrm{~h}$, but these effects were completely blocked by $5 \mu \mathrm{M}$ Compound $\mathrm{C}$ (Fig. 9c and d). These results further indicate that PGC-1 $\alpha$ expression is closely related to AMPK phosphorylation, and that the YQHX-induced activation of PGC- $1 \alpha$ expression may be attributed to the activation of AMPK phosphorylation in $\mathrm{H} 9 \mathrm{c} 2$ cells subjected to hypoxia.

\section{YQHX increased the expression of NRF-1 and Tfam in $\mathrm{H} 9 \mathrm{c} 2$ cells subjected to hypoxia}

Accumulating evidence indicate that PGC- $1 \alpha$ is a key controller of mitochondrial function through modulation of the nuclear respiratory factor (NRF-1) and the mitochondrial transcription factor A (Tfam) [21, 29]. A recent study showed that NRF-1 and Tfam expression were inhibited by Compound $\mathrm{C}$ in vivo [30]. Hence, we further investigated whether the protective effect of YQHX is related to NRF-1 and Tfam in H9c2 cells subjected to hypoxia. As shown in Fig. 10a, we observed that the expression of NRF-1 was significantly decreased in ischemia/hypoxia group compared with that of the control group $(P<0.05)$, whereas treatment with YQHX markedly increased NRF-1 expression $(P<0.05)$. This effect was completely blocked by Compound C. As shown in Fig. 10b, Tfam expression was lower in ischemia/hypoxia group compared with the control group $(P<0.05)$, while treatment with YQHX significantly increased Tfam expression $(P<0.05)$. Interestingly, this effect of YQHX was not blocked by Compound C. These results illustrate that the protective effects of YQHX on the mitochondrial are partly mediated through AMPK-dependent signaling pathways.

\section{Discussion}

IHD is characterized by the reduction of blood supply to the heart, leading to a series of events such as cardiomyocytes loss and ventricular remodeling all of which lead to heart failure [1]. Therefore, the discovery of effective therapeutic options for IHD is under intense research. Mitochondria are known to be the powerhouses in cardiomyocytes. During ischemia, mitochondria in the cardiomyocytes are the primary subcellular organelles affected by ischemia and are the major source of oxidative stress, leading a series of mitochondrial changes [5]. PGC$1 \alpha$, a transcriptional coactivator, acts as a master regulator of mitochondrial biogenesis and energy metabolism, and it also decreases oxidative stress [31], and promotes fatty acid oxidation and gluconeogenesis [32]. In this study, we investigated the expression of PGC- $1 \alpha$ in the ischemic myocardium. Particularly we investigated the effect of YQHX in cardiomyocytes subjected to hypoxic conditions. We demonstrated that PGC- $1 \alpha$ expression was suppressed following ischemic myocardial injury. The major findings of this study are as follows: (1) YQHX treatment might exert cardioprotective effects under ischemic conditions in vitro and vivo. (2) YQHX treatment could upregulate the expression of PGC- $1 \alpha$ in $\mathrm{MI}$ rats and hypoxia-induced $\mathrm{H} 9 \mathrm{c} 2$ cells, which improved the mitochondrial function. (3) YQHX treatment could improve mitochondrial structure and biosynthesis by increasing PGC-1 $\alpha, \mathrm{NRF}-1$ and Tfam protein expression in hypoxia-induced $\mathrm{H} 9 \mathrm{c} 2$ cells. The up-regulation of PGC-1 $\alpha$, NRF-1, and AMPK phosphorylation expression were completely blocked by AMPK inhibitor Compound C. (4) YQHX contains many active substances such as Astragaloside IV, Calycosin, Ferulic acid, Ginsenoside Rb1, Ginsenoside Rg3, Ginsenoside Rg1 and among others.

Using a myocardial infarction rat model, we firstly measured the LVEF and LVFS levels as representative parameters of cardiac function in rats MI model. These parameters were affected by myocardial infarction, while they were improved by YQHX and TMZ treatment (Fig. 3a). We then observed that YQHX restored the mitochondrial structural integrity and ATP production in the MI myocardium (Fig. 4). Furthermore, we showed that MI decreased the expression of PGC-1 $\alpha$, which was prevented by YQHX treatment. These results were consistent with the observations that PGC- $1 \alpha$ was downregulated under chronic hypoxia in pulmonary arterial hypertension [14] and respiratory muscles [33]. Our results supported the hypothesis that YQHX might exert cardioprotection by upregulating $\mathrm{PGC}-1 \alpha$ expression in vivo. However, the 


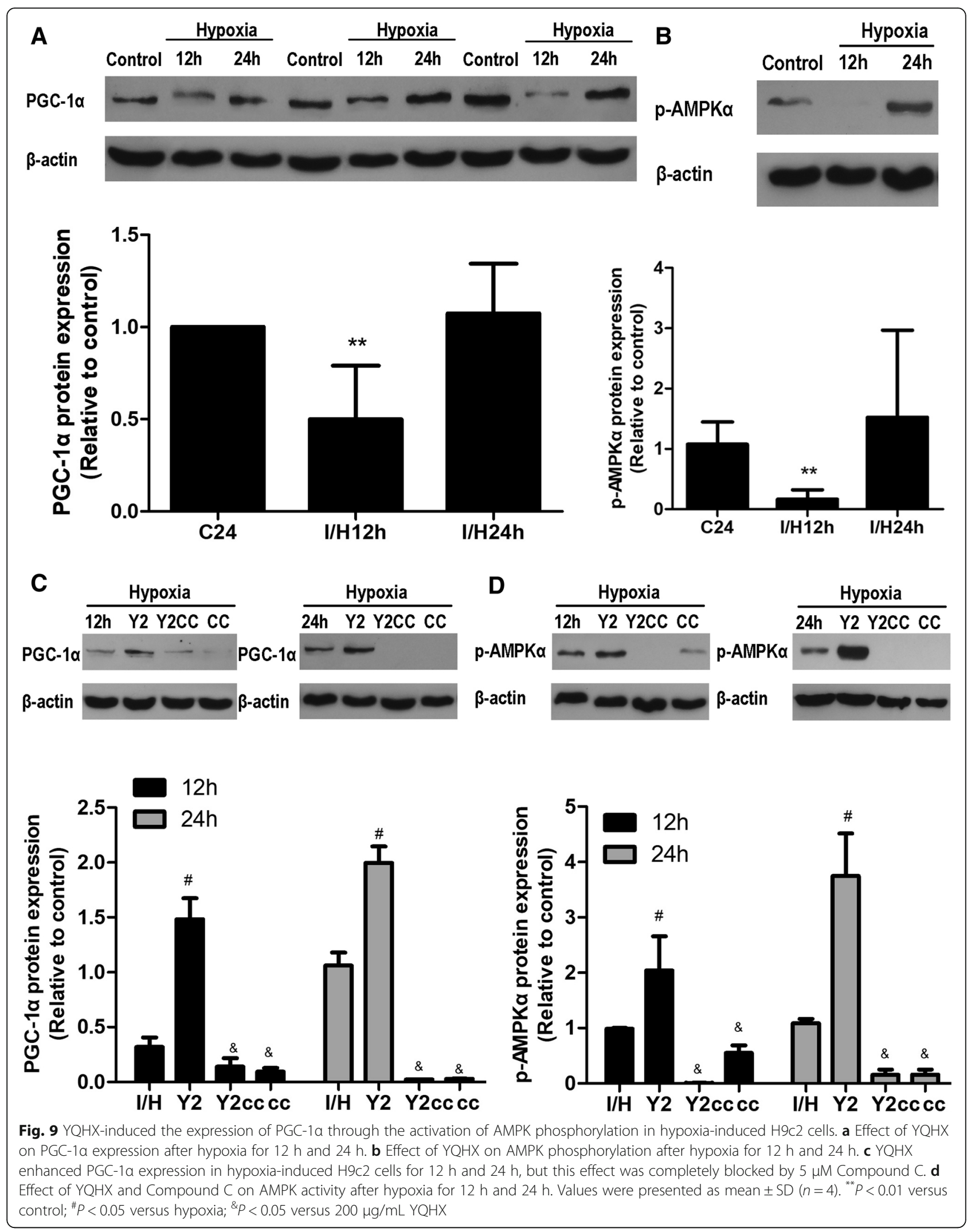


A
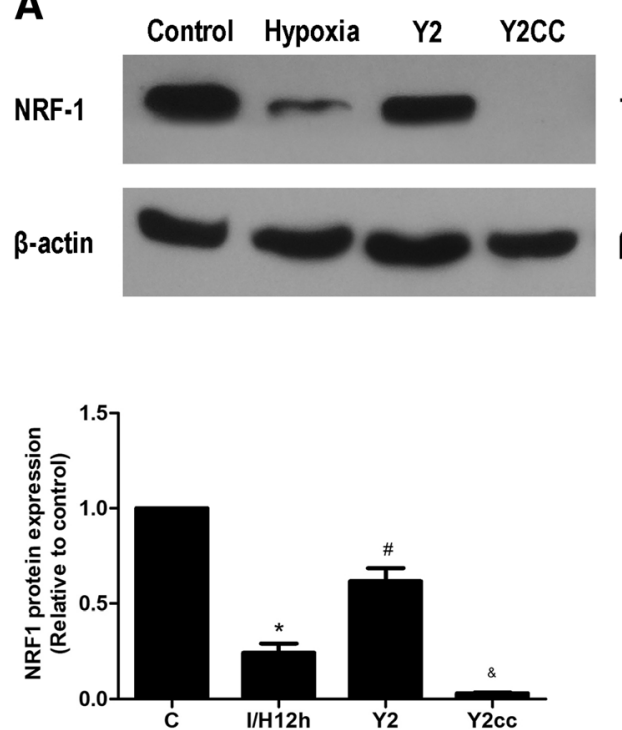

B

Tfam

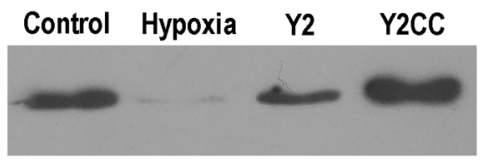

$\beta$-actin

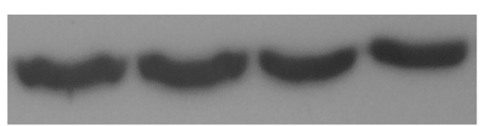

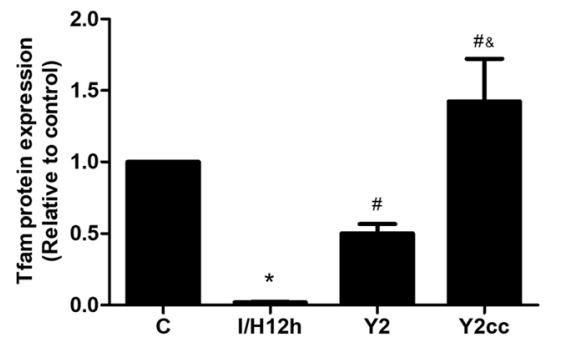

Fig. $10 \mathrm{YQHX}$ enhanced the expression of mitochondrial biogenesis molecules induced by hypoxia for $12 \mathrm{~h}$. a Effects of YQHX and Compound C on NRF-1 expression in hypoxia-induced H9c2 cells. $\mathbf{b}$ Tfam expression in hypoxia-induced H9c2 cells. Data were presented as mean \pm SD $(n=3)$. ${ }^{*} P<0.05$ versus control; ${ }^{\#} P<0.05$ versus hypoxia. ${ }^{\&} P<0.05$ versus $200 \mu \mathrm{g} / \mathrm{mL}$ YQHX. $P<0.05$ was considered to be statistically significant

mechanism through which YQHX regulated the PGC-1 $\alpha$ expression in the ischemic heart was not previously studied, especially in the cardiomyocytes.

In vitro, we developed the hypoxia injury model in $\mathrm{H} 9 \mathrm{c} 2$ cardiomyocytes. Then, we assessed cell viability and LDH release. In the present study, YQHX treatment $(100-400 \mu \mathrm{g} / \mathrm{mL})$ increased cell viability and reduced the LDH release in hypoxia-induced $\mathrm{H} 9 \mathrm{c} 2$ cells, suggesting that YQHX has cardio-protective effects on cardiomyocytes during $\mathrm{I} / \mathrm{H}$. Mitochondria are recognized as the major cellular source of ROS production and it also act as the powerhouses in cardiomyocytes. Hypoxia induced the downregulation of PGC- $1 \alpha$ expression, which may cause ROS overproduction and mitochondrial dysregulation, further exacerbating cardiomyocytes injury. Our data showed that hypoxia up-regulated ROS levels, decreased mitochondrial membrane potential, and disrupted the mitochondrial ultrastructures. Treatment with YQHX decreased ROS levels (Fig. 8d), improved cardiomyocytes mitochondrial ultrastructures and the depolarization of mitochondrial membrane potential, suggesting that YQHX has a protective role on the mitochondrial function in hypoxia-induced cardiomyocytes injury.

PGC- $1 \alpha$ is a key precursor of ROS production and extinction [14], which also acts as a powerful regulator of mitochondrial biosynthesis [29]. We also found that hypoxia decreased PGC- $1 \alpha$ mRNA and protein expression and damaged mitochondrial ultrastructures in $\mathrm{H} 9 \mathrm{c} 2$ cells subjected to hypoxia for $12 \mathrm{~h}$ (Fig. 8a-b), while $200 \mu \mathrm{g} / \mathrm{mL}$ YQHX significantly enhanced PGC- $1 \alpha$ mRNA and protein expression in hypoxia-induced H9c2 cells. Consistent with our results, hypoxia has been reported to decrease the PGC-1 $\alpha$ level in hepatocytes [34]. However, recent studies have demonstrated that PGC- $1 \alpha$ expression showed a different response at $12 \mathrm{~h}$ and $24 \mathrm{~h}$ of hypoxia in pulmonary artery endothelial cells [14]. Therefore, we investigated whether hypoxia for $12 \mathrm{~h}$ and $24 \mathrm{~h}$ affected the PGC- $1 \alpha$ expression in $\mathrm{H} 9 \mathrm{c} 2$ cardiomyocytes in a similar manner. Interestingly, PGC- $1 \alpha$ protein expression nearly returned to the normal level at $24 \mathrm{~h}$ of hypoxia (Fig. 9a), while YQHX markedly upregulated PGC- $1 \alpha$ expression compared to $24 \mathrm{~h}$ of hypoxia. Furthermore, the effects of hypoxia and YQHX on PGC- $1 \alpha$ expression were completely blocked by AMPK inhibitor Compound C (Fig. 9c). AMPK is a well-known serine-threonine kinase, which acts as an intracellular energy sensor. Thus, we subsequently assessed the phosphorylation of AMPK in hypoxia-stimulated $\mathrm{H} 9 \mathrm{c} 2$ cells. In agreement of with the changes of PGC- $1 \alpha$ expression, phosphorylation of AMPK at Thr172 was significantly decreased at $12 \mathrm{~h}$, but it showed an increasing trend at $24 \mathrm{~h}$ of hypoxia compared with the control group (Fig. 9b). Our results indicated that the mechanism of PGC- $1 \alpha$ expression occurred via AMPKmediated phosphorylation, and that YQHX-induced upregulation of PGC- $1 \alpha$ expression is closely linked to the activation of AMPK-mediated phosphorylation.

Accumulating evidences indicate that NRF-1 and Tfam are downstream targets of PGC- $1 \alpha$ that play a role in mitochondrial biogenesis and respiratory gene expression $[34,35]$. Therefore, we hypothesized that YQHX might 
regulate NRF-1 and Tfam to impact cardio-protection in cardiomyocytes. Indeed, our results showed that YQHX treatment upregulated NRF-1 and Tfam expression in hypoxia-induced H9c2 cells. The upregulation of NRF1 expression by YQHX was abrogated by Compound $\mathrm{C}$ (Fig. 10). However, Compound $\mathrm{C}$ in combination with YQHX treatment enhanced Tfam expression in hypoxiainduced $\mathrm{H} 9 \mathrm{c} 2$ cells compared with YQHX treatment alone. It is recognized that NRF-1 and Tfam expression can be inhibited by Compound $\mathrm{C}$ in vivo [30]. These results indicated that the protective effect of YQHX on H9c2 cells may be orchestrated through multiple targets. They also suggested that the mechanisms of YQHX on PGC- $1 \alpha$ expression in hypoxia-induced injury are complex, which point to the possibility that the other unidentified mechanisms contribute to the regulation of PGC- $1 \alpha$ expression. For example, silent information regulator of transcription SIRT1, a nutrient sensor through the $\mathrm{NAD}^{+}$ dependent histone deacetylation, may deacetylate multiple lysine residues in PGC- $1 \alpha$ which promotes mitochondrial fatty acid oxidation [9]. However, the mechanism by which YQHX regulates SIRT1 expression should be further studied.

Protective properties of YQHX have been widely described in the cardiovascular diseases, especially through ECG manifestations and assessment of left ventricular function in patients with myocardial infarction [19]. In rats MI model, our previous studies showed that YQHX regulated the metabolic-related products such as lipids, amino acids and glycolipids [20]. Also, YQHX was reported to ameliorate the cardiac energy metabolism via cross-talk between the LKB1-dependent Notch1 and AMPK after myocardial infarction [21]. However, the chemical constituents in YQHX have not been completely revealed. In this study, we identified 87 constituent compounds in YQHX extracts using the optimized LC-ESI-MS ${ }^{\mathrm{n}}$ method. Among the 87 compounds, Astragaloside IV (59), Calycosin (13), Ferulic acid (7), Ginsenoside Rg1 (43), Ginsenoside Rb1 (47), Ginsenoside Rg3 (80), Ginsenoside Rg5 (72), Ginsenoside Rd. (62), and Ginsenoside Re (24) have been reported to have multiple biological activities such as anti-inflammation, anti-apoptosis and anti-oxidative stress, which are related to cardiovascular diseases [3645]. For example, Astragaloside IV (ASIV, the major component of Astragalus membranaceus) was found to alleviate heart failure via regulating glucose and lipid metabolism and to attenuate isoproterenol-induced cardiac hypertrophy by mediating energy biosynthesis [36, 37]. Calycosin (the monomer of Astragalus membranaceus) was reported to improve left cardiac function in the myocardial infarction rats model [38]. Ferulic acid (FA, the monomer of Angelica sinensis) has been suggested to be a potential treatment for cardiovascular diseases [39]. Ginsenoside Rg1 (G-Rg1, the major constituent of Panax ginseng and Panax notoginseng) could modulate energy metabolism in rat myocardial ischemia/reperfusion injury [40] and Ginsenoside $\mathrm{Rb} 1$ (G-Rb1, the major ingredient of Panax ginseng) could decrease the myocardial infarct size and cardiac enzymes [41]. Ginsenoside Rg3 (G-Rg3, the effective components of Panax ginseng) could improve cardiac function by attenuating apoptosis and inflammation [42]. Ginsenoside Rg5 (G-Rg5, the monomer of Panax ginseng) could decrease cardiomyocytes apoptosis in isoproterenol-induced cardiac ischemia injury [43]. Ginsenoside Rd. (G-Rd, the monomer of Panax ginseng) was found to affect ischemia/reperfusion-induced oxidative stress [44]. Ginsenoside Re (G-Re, the monomer of Panax ginseng) was also found to have cardioprotective effects against cardiac ischemic and reperfusion injury [45]. The compounds identified in YQHX can be mainly classified into triterpenoid saponins and flavonoids, which mainly belong to Astragalus membranaceus and Panax ginseng. Our study showed that YQHX treatment significantly enhanced PGC- $1 \alpha$ expression in vivo and vitro. Consistent with our results, numerous compounds in YQHX extract were reported to increase PGC- $1 \alpha$ levels. For instance, Astragaloside IV could alleviate isoproterenolinduced myocardial hypertrophy by modulating NFkappaB/PGC-1alpha signaling in energy biosynthesis [37]. Ginsenoside Rb1 was reported to increase basal glucose uptake and to promote browning as evidenced by the increased UCP-1 and PGC- $1 \alpha$ mRNA expressions in 3 T3-L1 mature adipocytes [46, 47]. It was also reported that Ginsenoside $\operatorname{Rg} 3$ combined with aerobic exercise training enhanced PGC- $1 \alpha$ and NRF- 2 protein levels in cardiac muscle [48]. These findings indicated that the effects of YQHX on PGC- $1 \alpha$ expression in hypoxia-induced $\mathrm{H} 9 \mathrm{c} 2$ cells injury might be related to the active components contained in YQHX including Astragaloside IV, Ginsenoside Rb1, Ginsenoside Rg3, and Ginsenoside $\operatorname{Rg} 1$ as shown in Fig. 1.

\section{Conclusions}

In summary, the present findings provide evidence that PGC- $1 \alpha$ is suppressed in myocardial ischemic injury. Firstly, we confirm that $\mathrm{YQHX}$ has 87 chemical constituents using UHPLC-LTQ-Orbitrap-MS. YQHX treatment exerts cardioprotective effects in ischemic conditions both in vivo and vitro. YQHX treatment upregulates the PGC- $1 \alpha$ expression in $\mathrm{MI}$ rats and improves mitochondrial function. In vitro, YQHX has a pharmacological potential in the treatment of myocardial ischemic injury by upregulating cardiomyocytes PGC- $1 \alpha$, NRF-1, and Tfam expression. The regulation of PGC-1 $\alpha$ and NRF-1 expressions were blocked with AMPK inhibitor Compound $\mathrm{C}$. Therefore, the protective mechanism of YQHX on the myocardium through PGC- $1 \alpha$ may be due to the regulation of AMPK phosphorylation. 


\section{Additional file}

Additional file 1: Structure identification of chemical constituents of YQHX formula by UHPLC-LTQ Orbitrap MS. There were 87 peaks in YQHX preparations. These data provided valuable information, on the molecular weights and structure of the constituents. (DOCX $27 \mathrm{~kb}$ )

\section{Abbreviations}

CCK-8: Cell counting kit-8; ECG: Echocardiography; HE: Hematoxylin and eosin; HPLC-DAD-ESI-IT-TOF-MS ${ }^{n}$ : High performance liquid chromatographydiode array detection-electrospray ionization-hybrid ion trap-time of flightmass spectrometry; IHD: Ischemic heart diseases; PGC-1a: Peroxisome proliferator-activated receptor gamma co-activator; qRT-PCR: quantitative real-time polymerase chain reaction; TCM: Traditional Chinese medicine; TEM: Transmission electron microscopy; YQHX: Yiqihuoxue Decoction

\section{Acknowledgements}

All authors thank the research team members and Dongzhimen Hospital Affiliated to Beijing University of Chinese Medicine in the study.

\section{Funding}

This work was supported by the National Natural Science Foundation of China: Study of Influence of Supplementing Qi and Activating Blood Circulation Herbs on Mitochondrial Energy Metabolism and Signal Transduction of Myocardial Ischemia Rats (No. 81473552). The funding provides the financial support for the conduct of the research, and guides the design of this study about energy metabolism in myocardial infarction injury.

\section{Availability of data and materials}

We have presented all our main data in the form of figures and additional file. The datasets supporting the conclusions of this article are included within the article.

\section{Authors' contributions}

FL and SG conceived and designed the experiments; FL drafted the paper, SG and CW revised the paper; SG provided the funding and guided the design of the experiments; CW identified of Chemical Constituents; FL, XH and HW performed the experiments; XT, QC and JW produced animal model and measured the heart samples; $\mathrm{FL}, \mathrm{XH}$ and HW performed the cardiomyocyte experiments and measured the cardiomyocyte samples. JW, $Y Z$ and $X C$ performed analysis with the constructive discussions. $W L$ and $B Z$ assisted with the experimental design and animal experiments. All authors have read and approved the final manuscript for publication.

\section{Ethics approval}

In according with the Guide for the Animal Care and Use of Laboratory Animals published by the National Institutes of Health (NIH Publications No. 85-23, revised 1996), all experimental protocols and animal handling procedures were approved by the Animal Care Committee and Use Committee of Dongzhimen Hospital Affiliated to Beijing University of Chinese Medicine (2017-11)

\section{Consent for publication}

Not applicable.

\section{Competing interests}

The authors declare that they have no competing interests.

\section{Publisher's Note}

Springer Nature remains neutral with regard to jurisdictional claims in published maps and institutional affiliations.

\section{Received: 1 May 2018 Accepted: 2 September 2018} Published online: 17 September 2018

\section{References}

1. Makarewich CA, Zhang HY, Davis J, et al. Transient receptor potential channels contribute to pathological structural and functional remodeling after myocardial infarction. Circ Res. 2014;115:567-80.
2. Choi D, Hwang KC, Lee KY, et al. Ischemic heart diseases: current treatments and future. J Control Release. 2009;140:194-202.

3. GBD 2016 Causes of Death Collaborators. Global, regional, and national agesex specific mortality for 264 causes of death, 1980-2016: a systematic analysis for the Global Burden of Disease Study 2016. Lancet. 2017;390:1151-210.

4. Chandrasekhar J, Mehran R. The ideal anticoagulation strategy in STelevation myocardial infarction. Prog Cardiovasc Dis. 2015;58:247-59.

5. Pei HF, Hou JN, Wei FP, et al. Melatonin attenuates Postmyocardial infarction injury via increasing Tom70 expression. J Pineal Res. 2017;62:1.

6. Ong SB, Hall AR, Hausenloy DJ. Mitochondrial dynamics in cardiovascular health and disease. Antioxid Redox Signal. 2013;19:400-14.

7. Pei H, Yang Y, Zhao H, Li X, Yang D, Li D. The role of mitochondrial functional proteins in ROS production in ischemic heart diseases. Oxidative Med Cell Longev. 2016;2016:5470457.

8. Di Lisa F, Menabò R, Canton M, Barile M, Bernardi P. Opening of the mitochondrial permeability transition pore causes depletion of mitochondrial and cytosolic NAD ${ }^{+}$and is a causative event in the death of myocytes in Postischemic reperfusion of the heart. J Biol Chem. 2001;276:2571-5.

9. Scarpulla RC. Metabolic control of mitochondrial biogenesis through the PGC-1 family regulatory network. Biochim Biophys Acta. 1813;2011:1269-78.

10. Karunakaran D, Thrush AB, Nguyen MA, et al. Macrophage mitochondrial energy status regulates cholesterol efflux and is enhanced by anti-miR33 in atherosclerosis. Circ Res. 2015;117:266-78.

11. Kang D, Hamasaki N. Mitochondrial transcription factor a in the maintenance of mitochondrial DNA: overview of its multiple roles. Ann N Y Acad Sci. 2005;1042:101-8.

12. Gundewar S, Calvert JW, Jha S, et al. Activation of AMP-activated protein kinase by metformin improves left ventricular function and survival in heart failure. Circ Res. 2009;104:403-11.

13. Aatsinki SM, Buler M, Salomaki H, et al. Metformin induces PGC-1alpha expression and selectively affects hepatic PGC-1alpha functions. $\mathrm{Br} J$ Pharmacol. 2014:171:2351-63.

14. Ye JX, Wang SS, Ge M, et al. Suppression of endothelial PGC-1alpha is associated with hypoxia-induced endothelial dysfunction and provides a new therapeutic target in pulmonary arterial hypertension. Am J Physiol Lung Cell Mol Physiol. 2016;310:L1233-42.

15. Lin HQ, Gong AG, Wang HY, et al. Tsim Danggui Buxue tang (Astragali Radix and Angelicae Sinensis Radix) for menopausal symptoms: a review. J Ethnopharmacol. 2017;199:205-10.

16. Hu G, P Yang, Y Zeng, et al. Danggui Buxue decoction promotes angiogenesis by up-regulation of VEGFR1/2 expressions and downregulation of sVEGFR1/2 expression in myocardial infarction rat. J. Chin. Med. Assoc. 2018; 81, 37-46.

17. Mak DHF, Chiu PY, Dong TTX, et al. Dang-Gui Buxue tang produces a more potent cardioprotective effect than its component herb extracts and enhances glutathione status in rat heart mitochondria and erythrocytes. Phytother Res. 2006;20(7):561-7.

18. Guo SW, Yang PC, Zheng CL. Clinical research on the treatment of CHD myocardial ischemia-induced syndrome of qi-deficiency and blood stasis with TCM Yiqihuoxue herbs combined with conventional western medicine. Chin J Inform Traditional Chin Med. 2012;19(9):15-7.

19. Zheng $C L$, Yang PC, Zhang $L$, et al. Angiogenesis and expression of vascular endothelial growth factor and endostatin protein in myocardial infarction rat model. Bangladesh J Pharmacol. 2016;11:S1-7.

20. Zhang $L$, Chenglong Zheng, Min Jiang, et al. Effect of Yiqi Huoxue decoction on the metabolomics of acute myocardial infarction rats. J Traditional Chin Med Sci. 2017; 06, 001

21. Wu J G, X Chen, S W Guo, et al. Effect of Yiqihuoxue prescription on myocardial energy metabolism after myocardial infarction via cross talk of liver kinase B1-dependent Notchl and adenosine 5'-monophosphateactivated protein kinase. J Tradit Chin Med 2017; 37, 378-386.

22. Wu Jiangong, Guo Shuwen, Chen Xi, et al. YiqiHuoxue prescription can prevent and treat post-MI myocardial remodeling through promoting the expression of AMPK signal pathway. J Traditional Chin Med Sci 2017; 4(3), 235-244.

23. Arumugam $\mathrm{S}$, Sreedhar $\mathrm{R}$, Thandavarayan $\mathrm{RA}$, et al. Targeting fatty acid metabolism in heart failure: is it a suitable therapeutic approach? Drug Discov Today. 2016:21:1003-8.

24. Murphy E, Ardehali $H$, Balaban RS, et al. Mitochondrial function, biology, and role in disease: a scientific statement from the American Heart Association. Circ. Res. 2016;118:1960-91. 
25. Murphy E, Steenbergen C. Preconditioning: the mitochondrial connection. Annu Rev Physiol. 2007;69:51-67.

26. Li F, Guo S, Hu J, et al. Comparison on pharmacodynamics effects between different extracts of Yiqihuoxue on H9c2 myocardial cells injury induced by hypoxia. Guiding J Traditional Chin Med Pharm. 2018;24(3):29-32.

27. Ramjiawan A, Bagchi RA, Blant $A$, et al. Roles of histone deacetylation and AMP kinase in regulation of cardiomyocyte PGC-1alpha gene expression in hypoxia. Am J Physiol Cell Physiol. 2013;304:C1064-72.

28. Zhu L, Wang Q, Zhang L, et al. Hypoxia induces PGC-1alpha expression and mitochondrial biogenesis in the myocardium of TOF patients. Cell Res. 2010;20:676-87.

29. Chiang MC, Nicol CJ, Cheng YC, et al. Rosiglitazone activation of PPARgamma-dependent pathways is neuroprotective in human neural stem cells against amyloid-beta-induced mitochondrial dysfunction and oxidative stress. Neurobiol Aging. 2016;40:181-90.

30. Chung MM, Chen YL, Pei D, et al. The neuroprotective role of metformin in advanced glycation end product treated human neural stem cells is AMPKdependent. Biochim Biophys Acta. 1852;2015:720-31.

31. Xiao W, Goswami PC. Down-regulation of peroxisome proliferator activated receptor gamma coactivator 1alpha induces oxidative stress and toxicity of 1-(4-Chlorophenyl)-benzo-2,5-quinone in $\mathrm{HaCaT}$ human keratinocytes. Toxicol in Vitro. 2015;29:1332-8.

32. Handschin C, Spiegelman BM. Peroxisome proliferator-activated receptor gamma coactivator 1 coactivators, energy hemeostasis, and metabolism. Endocr Rev. 2006;27:728-35.

33. Gamboa JL, Andrade FH. Mitochondrial content and distribution changes specific to mouse diaphragm after chronic normobaric hypoxia. Am J Physiol Regul Integr Comp Physiol. 2010;298:R575-83.

34. Liu Y, Ma Z, Zhao C, et al. HIF-1alpha and HIF-2alpha are critically involved in hypoxia-induced lipid accumulation in hepatocytes through reducing PGC1alpha-mediated fatty acid beta-oxidation. Toxicol Lett. 2014;226:117-23.

35. Puigserver P, Spiegelman BM. Peroxisome proliferator-activated receptorgamma coactivator 1 alpha (PGC-1 alpha): transcriptional coactivator and metabolic regulator. Endocr Rev. 2003;24:78-90.

36. Dong Z, Zhao P, Xu M, et al. Astragaloside IV alleviates heart failure via activating PPARalpha to switch glycolysis to fatty acid beta-oxidation. Sci Rep. 2017;7(1):2691.

37. Zhang $S$, Tang F, Yang Y, et al. Astragaloside IV protects against isoproterenolinduced cardiac hypertrophy by regulating NF-kappaB/PGC-1alpha signaling mediated energy biosynthesis. PLoS One. 2015;10:e0118759.

38. Junqing $\mathrm{G}$, Tao C, Huigen J, et al. Effect of calycosin on left ventricular ejection fraction and angiogenesis in rat models with myocardial infarction. J Tradit Chin Med. 2015;35(2):160-7.

39. Mancuso C, Santangelo R. Ferulic acid: pharmacological and toxicological aspects. Food Chem Toxicol. 2014;65:185-95.

40. Li L, Pan CS, Yan L, et al. Ginsenoside Rg1 ameliorates rat myocardial ischemia-reperfusion injury by modulating energy metabolism pathways. Front Physiol. 2018;9:78.

41. Zheng Q, Bao XY, Zhu PC, et al. Ginsenoside Rb1 for myocardial ischemia/ reperfusion injury: preclinical evidence and possible mechanisms. Oxidative Med Cell Longev. 2017;2017:6313625.

42. Zhang $L P$, Jiang $Y C$, Yu XF, et al. Ginsenoside Rg3 improves cardiac function after myocardial ischemia/reperfusion via attenuating apoptosis and inflammation. Evid Based Complement Alternat Med. 2016;2016:6967853.

43. Yang YL, Li J, Liu K, et al. Ginsenoside Rg5 increases cardiomyocyte resistance to ischemic injury through regulation of mitochondrial hexokinase-II and dynamin-related protein 1. Cell Death Dis. 2017;8(2):e2625.

44. Zeng X, Li J, Li Z. Ginsenoside Rd mitigates myocardial ischemia-reperfusion injury via Nrf2/HO-1 signaling pathway. Int J Clin Exp Med. 2015;8(8):14497-504.

45. Lim KH, Lim DJ, Kim JH. Ginsenoside-re ameliorates ischemia and reperfusion injury in the heart: a hemodynamics approach. Journal of ginseng research. 2013;37(3):283-92.

46. Mu QQ, Fang X, Li XK, et al. Ginsenoside Rb1 promotes browning through regulation of PPAR gamma in 3T3-L1 adipocytes. Biochem Biophys Res Commun. 2015;466(3):530-5.

47. Liu JL, Zhang DW, Gao SH. Ginsenoside Rb1 promotes browning through regulation of PPAR gamma in 3T3-L1 adipocytes. Biochem Biophys Res Commun. 2015;466:530-5.

48. Sun M, Huang C, Wang C, et al. Ginsenoside Rg3 improves cardiac mitochondrial population quality: mimetic exercise training. Biochem Biophys Res Commun. 2013;441:169-74.

Ready to submit your research? Choose BMC and benefit from:

- fast, convenient online submission

- thorough peer review by experienced researchers in your field

- rapid publication on acceptance

- support for research data, including large and complex data types

- gold Open Access which fosters wider collaboration and increased citations

- maximum visibility for your research: over $100 \mathrm{M}$ website views per year

At BMC, research is always in progress.

Learn more biomedcentral.com/submissions 